\title{
Práticas integrativas e complementares para alívio ou controle da dor em oncologia
}

\section{Integrative and complementary practices for pain relieving or controlling in oncology}

\section{Ana Carolina de Abreu Moura' (D) Cíntia Carolina Silva Gonçalves ${ }^{2}$ (D)}

${ }^{1}$ Autora para correspondência. Centro Universitário Ruy Barbosa-UniRuy/Wyden (Salvador). Bahia, Brasil. ana.moura@hotmail.com.br

${ }^{2}$ Centro Universitário Ruy Barbosa-UniRuy/ Wyden (Salvador). Bahia, Brasil. cintia.goncalves@frb.edu.br

RESUMO I OBJETIVO: Apresentar as principais práticas integrativas e complementares (PICS) empregadas para alívio ou controle da dor em oncologia e identificar a práxis dos enfermeiros na utilização das PICS em pacientes oncológicos. MÉTODO: Revisão integrativa da literatura nas bases de dados LILACS, SciELO, BDENF e MEDLINE durante o período de 2008 a 2018. Utilizada a análise do conteúdo de Bardin. RESULTADOS: Foram selecionados 13 artigos para a realização deste estudo, sendo que $69,2 \%$ destes publicados por enfermeiros. Quanto ao ano de publicação, 2018 apresentou maior número de publicações $(30,8 \%)$ e a base de dados com mais publicações foi a LILACS $(53,8 \%)$. As principais PICS adotadas para o alívio ou controle da dor foram a acupuntura, acupuntura auricular, fitoterapia, reiki, homeopatia e a reflexologia podal. A práxis dos enfermeiros na utilização das PICS ressalta sua importância na educação em saúde e na sua aplicabilidade. CONCLUSÕES: As PICS, principalmente acupuntura, fitoterapia, reiki, homeopatia e reflexologia podal, representam recursos terapêuticos importantes no alívio da dor oncológica. A práxis dos enfermeiros na utilização desta terapêutica em pacientes oncológicos em hospitais, ainda se constitui um desafio em função da dificuldade de lidar com a hegemonia do modelo biomédico e à deficiência na formação acadêmico-profissional.

DESCRITORES: Práticas integrativas e complementares. Dor do câncer. Enfermagem. Neoplasias.

\begin{abstract}
OBJECTIVE: Presenting the main integrative and complementary practices used for pain reliefing or controlling in oncology and identifying nurses' praxis in the use of PICS in cancer patients. METHOD: Integrative literature reviewing in the LILACS, SCIELO, BDENF and MEDLINE databases from 2008 to 2018. Bardin content analysis was used. RESULTS: 13 articles were selected for this study. $69.23 \%$ of these were published by nurses. As for the year of publication, 2018 was the one with the most publications (30.76\%) and the database with the largest number of publications was LILACS (53.83\%). The main PICS adopted for pain relief or control were acupuncture, ear acupuncture, herbal medicine, reike, homeopathy and foot reflexology. The practice of nurses in the use of PICS highlights its importance in health education and its appplicability. CONCLUSIONS: Pain is a symptom experienced by most cancer patients, with which there is a wide field of action for nurses through PICS. Therefore, nurses must take ownership of PICS to make them integral and complementary to pharmacological therapies.
\end{abstract}

DESCRIPTORS: Integrative and complementary practices. Cancer pain. Nursing. Neoplasms. 


\section{Introdução}

O termo "câncer" é utilizado para designar doenças, cujo crescimento desordenado de células invade tecidos e órgãos, multiplicando-se de forma acelerada, descontrolada e agressiva. Tendo em vista a gravidade da doença, o Ministério da Saúde (MS), juntamente com o Instituto Nacional de Câncer (INCA), projetou uma estimativa de 600 mil novos casos de câncer durante o biênio 2018-2019. Já para cada ano do triênio 2020-2022, estima-se a incidência de 625 mil casos de câncer no Brasil ${ }^{1-3}$.

De acordo com o MS, o tratamento do câncer pode ser realizado por meio de uma ou até mesmo combinação de várias modalidades e técnicas, sendo elas: cirurgia oncológica, radioterapia, quimioterapia ou transplante de medula óssea³.

Segundo o INCA, a dor é um sintoma vivenciado por grande parte dos pacientes oncológicos, que corresponde de $46 \%$ a $92 \%$, dos acometidos pela patologia, e esse sintoma pode ser caracterizado da seguinte forma: relacionada diretamente ao câncer (12\% a $29 \%)$, ao tratamento antitumoral (5\% a $20 \%$ ) ou até mesmo pelas desordens concomitantes ( $8 \%$ a $22 \%)^{4}$.

A prevalência da dor tende a aumentar conforme a progressão do câncer, sendo vivenciada moderada ou intensa por $30 \%$ dos pacientes que estão fazendo tratamento e de $60 \%$ a $90 \%$ dos portadores em estágio avançado ${ }^{4}$. Por ser uma das queixas mais frequentes em pacientes oncológicos, a dor requer um tratamento adequado e intervenções complementares que visam reduzir o sofrimento relacionado a ela. Nesse contexto, as Práticas Integrativas e Complementares (PICS) podem ser aplicadas por enfermeiros ao avaliarem a dor e a resposta terapêutica, colaborando na reorganização do esquema farmacológico e propondo estratégias não farmacológicas para o alívio4.5.

As PICS são recursos terapêuticos que estimulam os mecanismos naturais de prevenção e recuperação da saúde por meio de tecnologias eficazes e seguras ${ }^{6}$. Em 2006, com a criação da Política Nacional de Práticas Integrativas e Complementares (PNPIC), foram inicialmente ofertadas, pelo Sistema Único de Saúde (SUS), 5 (cinco) PICS, porém, atualmente, 29 (vinte e nove) estão disponíveis para a população7-8.
Observa-se um amplo campo de atuação dos enfermeiros através das terapias complementares, devido a sua constante relação e convívio contínuo com o paciente, tornando importante o seu papel na implementação das práticas, a fim de que o alívio e/ou controle da dor ocorra. Em contrapartida, apesar das PICS serem recomendadas pelo Ministério da Saúde, pouca é a utilização destas pela enfermeira e mais reduzido ainda se constitui o quantitativo de publicações que abordem o trinômio enfermeira - dor - PICS 9 .

Baseando-se no exposto anteriormente, objetivou-se apresentar as principais práticas integrativas e complementares utilizadas para alívio ou controle da dor em oncologia e identificar a práxis dos enfermeiros na utilização das PICS.

\section{Método}

Trata-se de uma revisão integrativa da literatura, a qual tem sido apontada como uma ferramenta ímpar no campo da saúde, pois sintetiza as pesquisas disponíveis sobre determinada temática e direciona a prática fundamentando-se em conhecimento científico ${ }^{10}$.

Esta foi estruturada nas seis fases do processo de elaboração da revisão integrativa: elaboração da pergunta norteadora; busca ou amostragem na literatura; coleta de dados; análise crítica dos estudos incluídos; discussão dos resultados; e, apresentação da revisão integrativa ${ }^{10}$.

As buscas foram realizadas no portal Biblioteca Virtual em Saúde (BVS), filtrando os artigos dispostos nas bases de dados da Literatura Latino-Americana e do Caribe em Ciências de Saúde (LILACS), na Biblioteca Virtual Scientific Electronic Library Online (SciELO), na Base de Dados em Enfermagem (BDENF), Medical Literature Analysis and Retrieval System Online (MEDLINE).

A pesquisa nos Descritores em Ciências da Saúde (DeCS) possibilitou o levantamento e seleção de quatro descritores: "dor do câncer", "enfermagem", "neoplasias" e "terapias complementares". Após definição dos descritores e da palavra-chave, realizou-se uma combinação entre si por meio do conector booleano 
"AND". Sendo que o descritor "terapias complementares" foi utilizado isoladamente, ou seja, sem o uso do conector booleano "AND", na base de dados SciELO.

Os critérios de inclusão adotados para a seleção desta amostra foram: artigos originais e textos completos disponíveis na íntegra e online, que tivessem ligação com o tema proposto, publicados no idioma português no período de 2008 a 2018. Foram estabelecidos como critérios de exclusão os artigos repetidos nas bases de dados utilizadas e os artigos de revisão.

A análise dos dados foi baseada na análise do conteúdo de Bardin. Sendo dividida em 3 (três) estágios: pesquisa do material e organização conforme subtemas; exploração dos dados e posterior síntese dos aspectos importantes do texto; e, evidência e descrição das informações relevantes ${ }^{11}$.

Foram encontrados 5.994 artigos na Biblioteca Virtual em Saúde (BVS). Após aplicar os critérios de inclusão e exclusão adotados, 497 estudos foram identificados nas bases de dados e 438 excluídos após a leitura dos títulos e resumos por não atenderem ao objetivo desta pesquisa. Do total de 59 artigos completos selecionados, 46 foram excluídos após leitura na íntegra por não oferecerem subsídios para responder à pergunta norteadora, restando 13 artigos para realização da presente revisão.

A figura 1 abaixo ilustra o detalhamento do processo de escolha dos artigos selecionados para compor esta revisão integrativa.

Figura 1. Fluxograma da estratégia de busca dos artigos incluídos na revisão

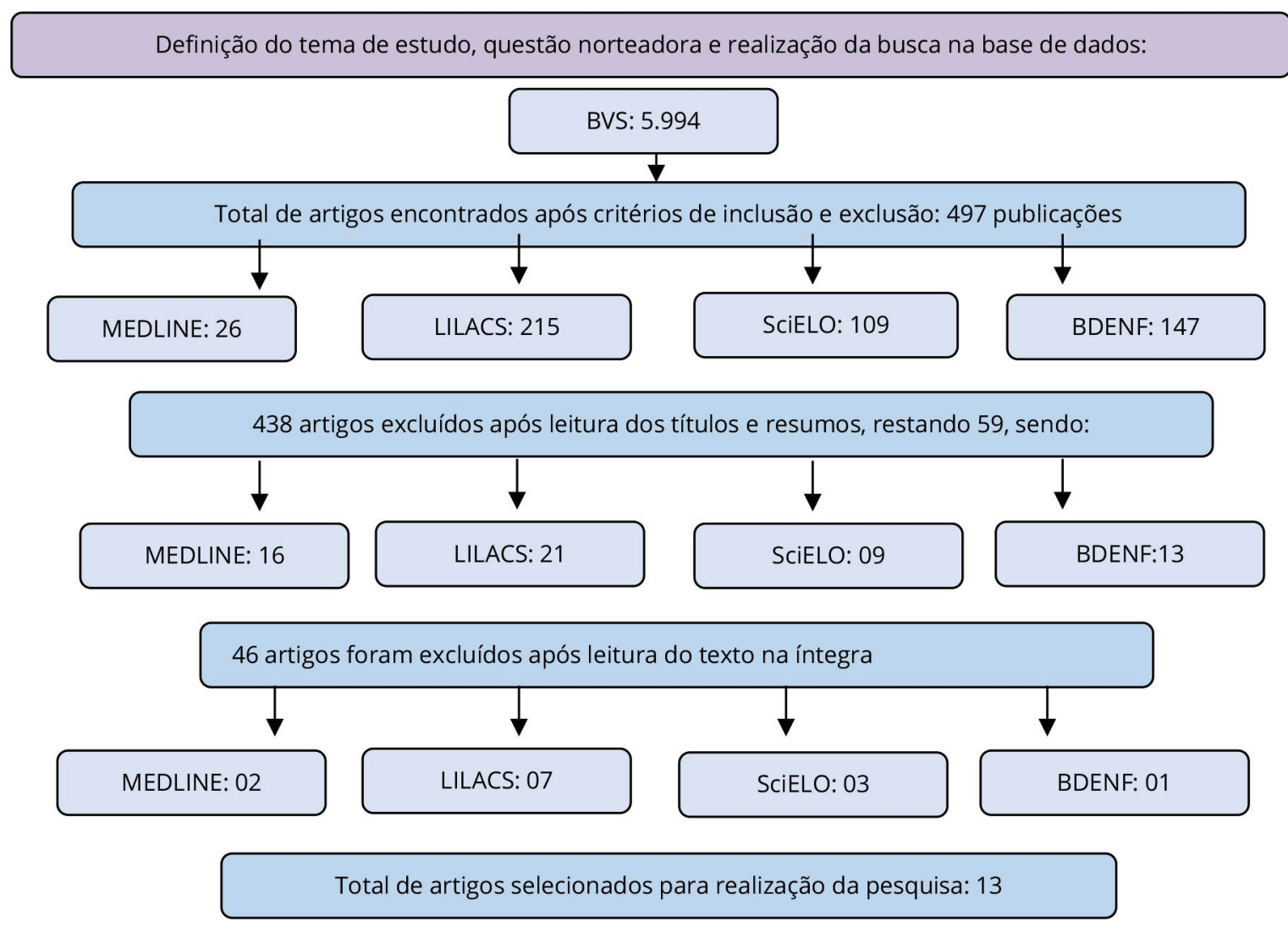

\section{Resultados e discussão}

Dos 13 artigos selecionados, quanto ao ano de publicação: 1 (um) artigo foi publicado em 2008, 2009, 2010 e 2012, correspondendo 7,69\% cada; 2 (dois) artigos (15,38\%) foram publicados em 2013; 3 (três) artigos (23,07\%) foram publicados em 2015; e, 4 (quatro) (30,76\%) no ano de 2018. 
A base de dados que mais publicou sobre a temática abordada foi a LILACS, correspondendo a 7 (sete) artigos, ou seja, 53,84\%. Foram identificados 69,23\%, representando 9 artigos realizados por enfermeiros.

Após a leitura detalhada das publicações selecionadas, foi realizada a síntese dos artigos com a confecção de um quadro contendo as seguintes informações: ano da publicação; base de dados; título do artigo; e, autores, com a finalidade de explicitar de forma padronizada as informações relevantes para esta revisão.

Quadro 1. Síntese dos artigos selecionados, em ordem decrescente

\begin{tabular}{|c|c|c|c|}
\hline $\begin{array}{c}\text { Ano da } \\
\text { Publicação }\end{array}$ & $\begin{array}{c}\text { Base de } \\
\text { Dados }\end{array}$ & Título & Autores \\
\hline 2018 & SCIELO & $\begin{array}{l}\text { Impactos das práticas integrativas e } \\
\text { complementares na saúde de } \\
\text { pacientes crônicos }\end{array}$ & Dacal MPO, Silva IS \\
\hline 2018 & SCIELO & $\begin{array}{l}\text { Efetividade da acupuntura auricular } \\
\text { no tratamento da dor oncológica: } \\
\text { ensaio clínico randomizado }\end{array}$ & $\begin{array}{l}\text { Ruela LO, lunes DH, Nogueira DA, } \\
\text { Stefanello J, Gradim CVC }\end{array}$ \\
\hline 2018 & LILACS & $\begin{array}{l}\text { Práticas integrativas complementares } \\
\text { na atenção primária à saúde }\end{array}$ & $\begin{array}{l}\text { Matos PC, Laverde CR, Martins PG, } \\
\text { Souza JM, Oliveira NF, Pilger C }\end{array}$ \\
\hline 2018 & LILACS & $\begin{array}{l}\text { Estratégias de ação e interação para o } \\
\text { cuidado à criança hospitalizada com } \\
\text { dor oncológica crônica }\end{array}$ & $\begin{array}{l}\text { Silva TP, Leite JL, Stinson J, Lallo C, } \\
\text { Silva IR, Jibb L }\end{array}$ \\
\hline 2015 & LILACS & $\begin{array}{l}\text { Plantas medicinais utilizadas pela } \\
\text { população do município de Lagarto- } \\
\text { SE, Brasil- ênfase em pacientes } \\
\text { oncológicos }\end{array}$ & $\begin{array}{l}\text { Caetano NLB, Ferreira TF, Reis } \\
\text { MRO, Neo GGA, Carvalho AA }\end{array}$ \\
\hline 2015 & LILACS & $\begin{array}{l}\text { Condutas terapêuticas utilizadas no } \\
\text { manejo da dor em oncologia }\end{array}$ & $\begin{array}{l}\text { Pereira DTS, Andrade LL, } \\
\text { Agra G, Costa MML }\end{array}$ \\
\hline 2015 & LILACS & $\begin{array}{l}\text { Problemas éticos vivenciados por } \\
\text { enfermeiros oncológicos }\end{array}$ & $\begin{array}{l}\text { Luz KR, Vargas MAO, Schmidtt PH, } \\
\text { Barlem ELD, Barlem JGT, Rosa LM }\end{array}$ \\
\hline 2013 & SciELO & $\begin{array}{l}\text { Práticas integrativas e } \\
\text { complementares no cuidado de } \\
\text { enfermagem: um enfoque ético }\end{array}$ & Magalhães MGM, Alvim NAT \\
\hline 2013 & MEDLINE & $\begin{array}{l}\text { Práticas complementares de saúde e } \\
\text { os desafios de sua aplicabilidade no } \\
\text { hospital: visão de enfermeiros }\end{array}$ & $\begin{array}{l}\text { Melo SCC, Santana RG, Santos DC, } \\
\text { Alvim NAT }\end{array}$ \\
\hline 2012 & BDENF & $\begin{array}{l}\text { Falando sobre câncer de colo uterino: } \\
\text { contribuições das terapias } \\
\text { complementares }\end{array}$ & $\begin{array}{l}\text { Melo MCP, Moura RG, Bezerra } \\
\text { MWS, Barroa AGS, Salum RDL, } \\
\text { Gomes LMA }\end{array}$ \\
\hline 2010 & LILACS & $\begin{array}{l}\text { Dialogando com enfermeiras sobre a } \\
\text { avaliação da dor oncológica do } \\
\text { paciente sob cuidados paliativos }\end{array}$ & $\begin{array}{l}\text { Waterkemper R, Reibnitz KS, } \\
\text { Monticelli M }\end{array}$ \\
\hline 2009 & MEDLINE & $\begin{array}{l}\text { Enfermidades tratadas e tratáveis pela } \\
\text { acupuntura segundo percepção de } \\
\text { enfermeiras }\end{array}$ & $\begin{array}{l}\text { Kurebayashi LFS, Freitas GF, } \\
\text { Oguisso T }\end{array}$ \\
\hline 2008 & LILACS & $\begin{array}{l}\text { A utilização de terapias alternativas } \\
\text { por pacientes em tratamento } \\
\text { quimioterápico }\end{array}$ & $\begin{array}{l}\text { Jaconodino CB, Amestoy SC, } \\
\text { Thofehrn MB }\end{array}$ \\
\hline
\end{tabular}


Após a análise dos artigos selecionados e para melhor compreensão dos resultados, viabilizou-se o agrupamento em 2 (duas) categorias: Práticas integrativas e complementares utilizadas na dor em oncologia; e, os enfermeiros e a práxis junto as PICS.

\section{Práticas integrativas e complementares utiliza- das na dor em oncologia}

A medicina tradicional chinesa, a exemplo, a acupuntura e a acupuntura auricular são indicadas e utilizadas como terapêutica para diversos tipos de dores ${ }^{12,13}$. Através de um ensaio clínico randomizado com 31 portadores de câncer que apresentavam queixa de dor, realizou uma comparação entre o grupo experimental que fez uso da acupuntura auricular e o grupo placebo para avaliar a efetividade da diminuição da dor e sua relação com a redução das doses diárias de analgésicos, após concluiu que, além da baixa na intensidade da dor, houve diferença significativa nas avaliações relacionadas ao número de analgésicos consumidos ${ }^{13}$.

Já a fitoterapia é a terapia de escolha entre as PICS utilizadas por pessoas portadoras do câncer, sendo o critério de escolha principal a questão cultural, o fácil acesso às plantas ou até mesmo o baixo custo para aquisição $14,15,16$.

Mediante entrevista com mulheres portadoras de câncer do colo uterino, avaliaram a contribuição das terapias complementares, e com os dados coletados, concluíram que a fitoterapia proporcionou um senso de autocontrole, revelando-se como a escolha principal dentre as PICS e como alternativa para o bem-estar das entrevistadas ${ }^{17}$.

Ao pesquisar as Plantas Medicinais (PM), dando ênfase ao seu uso por pacientes oncológicos no município de Lagarto-SE, revelou que $40 \%$ dos entrevistados utilizam PM concomitante com quimioterapia ${ }^{14}$.

Vale ressaltar que a fitoterapia deve ser utilizada de forma racional e com a devida orientação médi$\mathrm{ca}$, pois, quando usada pelos pacientes oncológicos concomitantes com quimioterapia, existe o risco de interações medicamentosas entre a planta medicinal e o antineoplásico ${ }^{14}$. Diante dessa situação, torna-se importante a avaliação e orientação de um profissional de saúde que tenha conhecimento sobre a Prática
Integrativa e Complementar (PIC) em questão, ressaltando a atuação do enfermeiro, como parte do processo de cuidado da saúde.

Neste estudo a maioria dos entrevistados (89\%) respondeu que são favoráveis à utilização das PICS em tratamento quimioterápico, e $69 \%$ fazem uso de alguma PIC, sendo elas a fitoterapia (82\%), a homeopatia (11\%) e reiki (7\%). Menos da metade (44\%) afirmaram que houve diminuição dos efeitos colaterais da quimioterapia e $40 \%$ também confessaram alívio nos sintomas. Finaliza, enfatizando que a "condição socioeconômica baixa e o valor acessível são fatores contribuintes para optar pelo uso da fitoterapia"16.

A dor no corpo foi a principal queixa relatada pelos pacientes, correspondendo a $85 \%$, sendo que $51 \%$ relataram melhora deste e outros sintomas, através do uso concomitante das práticas de reiki e a reflexologia podal ${ }^{18}$.

A PIC, denominada reiki, não foi identificada na PNPIC como sendo uma prática direcionada para a dor, porém pode ser utilizada de forma simultânea com outra PIC para promover o efeito energético e proporcionar o bem-estar físico e mental ${ }^{16,18}$.

Com o objetivo de identificar as principais modalidades terapêuticas utilizadas pela equipe de enfermagem, observa-se em 50 pacientes oncológicos, que fazem tratamento para dor, que além de utilizarem as terapias farmacológicas, 32\% desses afirmaram ter feito uso de terapêuticas não farmacológicas como alternativas para o alívio da dor. Entretanto, o tratamento farmacológico ainda predomina, seguindo a tendência do modelo biomédico, deixando de lado, muitas vezes, os aspectos biopsicossociais ${ }^{19}$.

A PNPIC traz o conceito, bem como as indicações das PICS, permitindo com isso, a identificação da hipnoterapia, que se caracteriza pelo uso de técnicas que pode ser associada a outra PIC, possibilitando o relaxamento intenso e permitindo o alívio de dores crônicas. Traz também a ozonioterapia, uma PIC bastante empregada na oncologia e que se utiliza da aplicação de dois gases (oxigênio e o ozônio) para promover o tratamento de algumas doenças e, consequentemente o alívio da dor. Todavia, não foram encontrados artigos nacionais que abordassem a relação das duas práticas no alívio ou controle da dor em pacientes oncológicos ${ }^{6}$. 


\section{Os enfermeiros e a práxis junto às PICS}

Constitui-se um grande desafio para a aplicabilidade das PICS (reiki, shiatsu, acupuntura, fitoterapia, musicoterapia, florais e cromoterapia) pelos enfermeiros em hospitais públicos, em função da dificuldade de lidar com a hegemonia do modelo biomédico nos hospitais atuantes. Outro fator que dificulta a utilização das PICS nos hospitais é a formação acadêmico-profissional, que ainda prevalece o domínio da prática convencional farmacológica ${ }^{15}$.

Com o objetivo de analisar o conhecimento e a percepção de 10 enfermeiras que atuam na atenção primária à saúde, em município goiano, 50\% relataram que conhecem as PICS, $100 \%$ reconhecem a importância dela relacionada à saúde, porém todas as entrevistadas disseram não conhecer uma Estratégia Saúde da Família (ESF) que oferte as PICS à população. Metade das enfermeiras entrevistadas relataram utilizar ou conhecer alguém que utilize as PICS e todas concordaram e acreditam sobre os efeitos benéficos obtidos com uso das práticas integrativas. Afirmaram ainda que as PICS mais incorporadas, conhecidas pela comunidade ou utilizadas por elas são a homeopatia, fitoterapia e a acupuntura ${ }^{20}$.

A importância do enfermeiro no seu papel educativo, através do diálogo com o paciente, orientando e elucidando as dúvidas acerca do diagnóstico e tratamento, bem como, ouvindo a manifestação da sua decisão, tornam-se fatores determinantes para a qualidade do cuidado ao paciente com câncer. Importante sinalizar os princípios da integralidade e da autonomia, ou seja, a capacidade do ser humano de decidir sobre algo, de acordo com seus valores, expectativas, necessidades, prioridades e $\operatorname{crenças~}^{21,22}$.

Em relação ao cuidado da enfermagem, através das PICS, as autoras citaram a reflexão e a reivindicação da legitimação no âmbito do cuidado de enfermagem. Falam, também, sobre a importância do diálogo paciente-enfermeiro acerca da finalidade e emprego correto das terapias para servir como coadjuvantes, tratamento e manutenção da saúde ${ }^{22}$.

Observaram que existe a necessidade de uma estruturação sistemática para o cuidado ao paciente oncológico que sofre com algia e chegaram a conclusão, em seu estudo realizado com a equipe de saúde, entre eles, enfermeiros e técnicos de enfermagem, em um hospital, na cidade do Rio de Janeiro, sobre a necessidade dos profissionais de saúde estabelecerem estratégias de ação que melhorem a interação com a criança hospitalizada, que apresenta dor oncológica, e seu familiar, para com isso facilitar o processo de avaliação e manejo da dor ${ }^{23,24}$.

A dificuldade da assistência integral fragmenta o cuidado ao ser humano, se distanciando do cuidado em si à pessoa, ressaltando, assim, a importância da sistematização desse cuidado direcionado para a avaliação, parâmetros avaliativos, controle e registro da dor pelo enfermeiro, promovendo a integralidade do seu atendimento ao paciente que realiza o tratamento da dor ${ }^{15,23}$.

\section{Conclusões}

As principais PICS utilizadas para o alívio ou controle da dor em oncologia são: a Acupuntura, Acupuntura auricular, a fitoterapia, o reiki, a homeopatia e a reflexologia podal.

Conclui-se também que essas práticas estão em constante evolução e aceitação por parte dos pacientes oncológicos que sofrem com a dor. Entretanto, por se tratar de uma política nacional relativamente nova, que regulamenta a sua atividade para toda a população, acredita-se que há muito a evoluir para uma maior aceitabilidade e conhecimento pelos profissionais de saúde e os pacientes.

A práxis dos enfermeiros na utilização das PICS ressalta a sua importância na aplicabilidade das PICS e na educação em saúde aos pacientes para que assim se estabeleça uma relação terapêutica, troca de conhecimentos, e os mesmos possam exercem a sua autonomia, ou seja, liberdade de decisão.

Portanto, é de suma importância que os enfermeiros se apropriem das PICS, para compreender a sua importância e torná-la de uso integral e complementar às terapias farmacológicas, difundindo o conhecimento e os resultados obtidos decorrente do seu uso. Bem como, enfrentando os desafios e dificuldade de lidar com a hegemonia do modelo biomédico e da prática convencional farmacológica. 
Recomenda-se novos estudos a respeito das PICS, visto que o pouco quantitativo de artigos publicados, referente a tais práticas relacionadas à dor seja ela oncológica ou não, configura como uma limitação na realização deste trabalho.

\section{Contribuições das autoras}

Moura ACA participou da concepção, delineamento, busca, análise dos dados da pesquisa, interpretação dos resultados. Gonçalves CCS participou como orientadora do artigo, revisando e aprovando a final da versão a ser publicada.

\section{Conflitos de interesses}

Nenhum conflito financeiro, legal ou político envolvendo terceiros (governo, empresas e fundações privadas, etc.) foi declarado para nenhum aspecto do trabalho submetido (incluindo, mas não se limitando a subvenções e financiamentos, participação em conselho consultivo, desenho de estudo, preparação de manuscrito, análise estatística, etc.).

\section{Referências}

1. Ministério da Saúde. Instituto Nacional de Câncer. Estimativa de Câncer no Brasil. [Internet]. 2019. [acesso em 2020 fev 10]. Disponível em: https://www.inca.gov.br/estimativa/introducao

2. Ministério da Saúde. Instituto Nacional de Câncer. Estimativa de câncer no Brasil. [Internet]. 2018. [acesso em 2020 fev 10]. Disponível em: https://www.inca.gov.br/en/node/2081

3. Ministério da Saúde. Câncer: o que é, causas, tipos, sintomas, tratamentos, diagnóstico e prevenção. [Internet]. 2019. [acesso em 2019 abr 9]. Disponível em: http://portalms.saude.gov.br/ saude-de-a-z/cancer

4. Ministério da Saúde. Instituto Nacional de Câncer. Cuidados paliativos oncológico: controle da dor. [Internet]. 2001. [acesso em 2019 abr 9]. Disponível em: http://bvsms.saude.gov.br/bvs/ publicacoes/inca/manual_dor.pdf

5. Graner KM, Costa Junior AL, Rolim GS. Dor em oncologia: intervenções complementares e alternativas ao tratamento medicamentoso. Temas em Psicologia. 2010;18(2):345-355.

6. Ministério da Saúde. Ministério da Saúde inclui 10 novas práticas integrativas no SUS. [Internet]. 2018. [acesso em 2018 mar 22]. Disponível em: http://portalms.saude.gov.br/noticias/ agencia-saude/42737-ministerio-da-saude-inclui-10-novaspraticas-integrativas-no-sus
7. Ministério da Saúde. Política Nacional de Práticas Integrativas e Complementares. [Internet]. [acesso em 2019 abr 9]. Disponível em: http://portalms.saude.gov.br/acoes-e-programas/politicanacional-de-praticas-integrativas-e-complementares-pnpic

8. Ministério da Saúde. Práticas Integrativas e Complementares (PICS): quais são e para que servem. [Internet]. 2017. [acesso em 2019 mai 6]. Disponível em: http://portalms.saude.gov.br/saudede-a-z/praticas-integrativas-e-complementares

9. Eler GJ, Jaques AE. O enfermeiro e as terapias complementares para o alívio da dor. Arquivos de Ciências da Saúde da Unipar. 2008;10(3):185-90. doi: 10.25110/arqsaude.v10i3.2006.624

10. Souza MT, Silva MD, Carvalho R. Revisão integrativa: o que é e como fazer. Einstein. 2010;8(1):102-6. doi: 10.1590/s167945082010rw1134

11. Bardin L. Análise de conteúdo. São Paulo: Edições 70; 2011.

12. Kurebayashi LFS, Freitas GF, Oguisso T. Enfermidades tratadas e tratáveis pela acupuntura segundo percepção de enfermeiras. Rev Esc Enferm. 2009;43(4):930-6. doi: 10.1590/ S0080-62342009000400027

13. Ruela LO, lunes DH, Nogueira DA, Stefanello J, Gradim CVC. Efetividade da acupuntura auricular no tratamento da dor oncológica: ensaio clínico randomizado. Rev Esc Enferm. 2018;52:e03402. doi: 10.1590/s1980-220×2017040503402

14. Caetano NLB, Ferreira TF, Reis MRO, Neo GGA, Carvalho AA. Plantas medicinais utilizadas pela população do município de Lagarto- SE, Brasil - ênfase em pacientes oncológicos. Rev Bras Plantas Med. 2015;17(4 supl 1):748-56. doi: 10.1590/1983084X/14_056

15. Melo SCC, Santana RG, Santos DC, Alvim NAT. Práticas complementares de saúde e os desafios de sua aplicabilidade no hospital: visão de enfermeiros. Rev Bras Enferm. 2013;66(6):84046. doi: $10.1590 / 50034-71672013000600005$

16. Jaconidino CB, Amestoy SC, Thofehrn MB. A utilização de terapias alternativas por pacientes em tratamento quimioterápico. Cogitare Enfermagem. 2008;13(1):61-6.

17. Melo MCP, Moura RG, Bezerra MWS, Barros AG, Salum RDL, Gomes LMA. Falando sobre câncer de colo uterino: contribuições das terapias complementares. Rev Pesqui Cuid Fundam. 2012;4(4):2909-19.

18. Dacal MPO, Silva IS. Impactos das práticas integrativas e complementares na saúde de pacientes crônicos. Saúde debate. 2018;42(118):724-735. doi: 10.1590/0103-1104201811815

19. Pereira DTS, Andrade LL, Agra G, Costa MML. Condutas terapêuticas utilizadas no manejo da dor em oncologia. Rev Pesqui Cuid Fundam. 2015;7(1):1883-1890. doi: 10.9789/21755361.2015.v7i1.1883-1890 
20. Matos PC, Laverde CR, Martins PG, Souza JM, Oliveira NF, Pilger C. Práticas integrativas complementares na atenção primária à saúde. Cogitare enferm. 2018;23(2): e54781. doi: 10.5380/ce.v23i2.54781

21. Luz KR, Vargas MAO, Schmidtt PH, Barlem ELD, Rosa LM. Problemas éticos vivenciados por enfermeiros oncológicos. Rev Latino-Am Enfermagem.2015;23(6):1187-94. doi: 10.1590/01041169.0098 .2665

22. Magalhães MGM, Alvim NAT. Práticas integrativas e complementares no cuidado de enfermagem: um enfoque ético. Esc Anna Nery. 2013;17(4):646-653. doi: 10.5935/1414$\underline{8145.20130007}$

23. Waterkemper R, Reibnitz KS, Monticelli M. Dialogando com enfermeiras sobre a avaliação da dor oncológica do paciente sob cuidados paliativos. Rev Bras Enferm. 2010;63(2):334-9. doi: 10.1590/S0034-71672010000200026

24. Silva TP, Leite JL, Stinson J, Lalloo C, Silva IR, Jibb L. Estratégias de ação e interação para o cuidado à criança hospitalizada com dor oncológica crônica. Texto Contexto Enferm.

2018;27(4):e3990017. doi: 10.1590/0104-07072018003990017 